\title{
High-Throughput Sequencing Reveals the Gut Microbiome of the Bactrian Camel in Different Ages
}

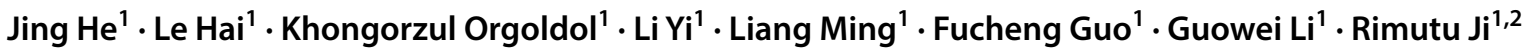

Received: 21 January 2019 / Accepted: 10 April 2019 / Published online: 27 April 2019

(c) The Author(s) 2019

\begin{abstract}
The complex gut microbiota plays a key role in host metabolism and health. However, the core microbial communities in the different aged Bactrian camels remain totally unclear. We used high-throughput 16S rRNA gene sequencing to examine the temporal variability of the fecal microbiota in Bactrian camels. At 2 months of age, the fecal microbiota was composed of Firmicutes, Proteobacteria, and Actinobacteria. At 1 and 3 years of age, the fecal microbiota was dominated by Firmicutes, Bacteroidetes, and Verrucomicrobia. At the genus level, Blautia, Fusobacterium, and Bifidobacterium were more abundant at 2 months of age, as well as Escherichia-Shigella. Ruminococcaceae_UCG-005, Akkermansia, and Christensenellaceae_R-7_ group were the most abundant at 1 and 3 years of age. Diversity and stability of the gut microbiota increased with age. There was enrichment for genes associated with immune system diseases at 2 months of age. This study is the first to investigate the distribution of the gut microbiota in Bactrian camels with different ages and provide a baseline for future camel microbiology research.
\end{abstract}

\section{Introduction}

The establishment of a stable gut microbiota is closely correlated with host growth and immune development. The gut microbiota is an important factor for mammalian health, and plays a critical role in metabolism, immunity, and host development [1,2]. A stable commensal community protects the host against invasive pathogens [3]. Microbial colonization of the infant begins at birth and is impacted by lactation $[4,5]$. The gut microbiota in the ruminants not only regulates body health but also plays an important bridging role between diet and host [6]. It has demonstrated that ruminants have unique digestive properties and microbial which can help host to adapt to high fiber content foods, but also

Electronic supplementary material The online version of this article (https://doi.org/10.1007/s00284-019-01689-6) contains supplementary material, which is available to authorized users.

Rimutu Ji

yeluotuo1999@vip.163.com

1 Key Laboratory of Dairy Biotechnology and Bioengineering, Ministry of Education, College of Food Science and Engineering, Inner Mongolia Agricultural University, Hohhot 010018, Inner Mongolia, China

2 Camel Research Institute of Inner Mongolia, Alxa 737300 , Inner Mongolia, China can make ruminants susceptible to a variety of diseases and conditions [7]. The gut microbiota in ruminants play a more prominent role in various physiological states [8].

The Bactrian camel inhabits the cold deserts of southern areas of central (Kazakhstan, Iran) and eastern (Russia, Mongolia, China) Asia [9]. The camel plays a vital role in the socio-economic of the region and is relied upon by millions of humans in both the semi-dry and arid areas. Previous studies also identified the species composition of the camel's rumen microbiome [10] and a detailed profiling of the camel rumen's carbohydrate-active enzymes [11]. Moreover, the gut microbiota may play a critical role in Bactrian camel health [12]. Microbial colonization begins at birth and is essential for the maintenance of host health. The composition of the early life microbiota has been studied in rats [13], cattle [14], and mice [15]. Furthermore, some potential links may also exist between age factors and intestinal microbiota. However, the dynamic shifts of the gut microbiota in Bactrian camels remain unclear. We used high-throughput 16S rRNA gene sequencing to investigate the microbiota composition of fecal samples from Bactrian camels. The results suggested that age is also important for shaping the Bactrian camel gut microbiota composition and contributing to its dynamic shifts. 


\section{Materials and Methods}

\section{Animals and Sample Collection}

Samples were collected from 18 Bactrian camels in Inner Mongolia Bayannaoer in the spring of 2018. There were three groups: 2-month-old camels that were fed breast milk (TM); 1-year-old camels that were fed a plant-based diet consisting of Chenopodiaceae, Compositae, and Leguminosae $(\mathrm{ON})$; and 3-year-old camels that were fed a plantbased diet consisting of Chenopodiaceae, Compositae, and Leguminosae (TH). Fresh fecal samples were immediately frozen using liquid nitrogen and were stored at $-80^{\circ} \mathrm{C}$. The experiment was conducted according to the animal ethics guidelines of the Key Laboratory of Dairy Biotechnology and Bioengineering, and approved by the Animal Ethics Committee of Inner Mongolia Agricultural University.

\section{DNA Extraction and 16S rRNA Gene Sequencing}

Genomic DNA was extracted using the QIAamp DNA stool mini kit (QIAGEN, cat\#51504), according to the manufacturer's protocol. The V3-V4 hypervariable region of the $16 \mathrm{~S}$ rRNA gene was amplified using primers the $515 \mathrm{~F}$ and 806R primers (ACTCCTACGGGAGGCAGCA and GGA CTACHVGGGTWTCTAAT, respectively). The reaction conditions were as follows: an initial denaturation at $98^{\circ} \mathrm{C}$ for $2 \mathrm{~min}$; followed by 30 cycles of denaturation at $98^{\circ} \mathrm{C}$ for $15 \mathrm{~s}$, annealing at $55^{\circ} \mathrm{C}$ for $30 \mathrm{~s}$, extension at $72{ }^{\circ} \mathrm{C}$ for $30 \mathrm{~s}$; and a final extension at $72{ }^{\circ} \mathrm{C}$ for $5 \mathrm{~min}$. The concentration and purity of DNA were tested using the Quant-iTTMPicoGreen ${ }^{\circledR}$ dsDNA Assay Kit (Life Technologies, Grand Island, NY, USA). Library preparation and sequencing were conducted by the Personal Biotechnology Co., Ltd. (Shanghai, China).

\section{Data Analysis}

The QIIME (Qiime1.8.0) was used for 16S rRNA data quality control and analysis [16]. We used the SILVA database (SILVA, Release 119) to analyze taxonomy [17]. The highquality sequences were used in the final analysis. Sequences were clustered into operational taxonomic units (OTUs) using the UCLUST algorithm (97\% similarity) in QIIME v.1.8.0. The Ribosomal Database Program (RDP) classifier was used to assign taxonomic category to all OTUs at a confidence threshold of 0.8. Alpha (Shannon, Chao1, Simpson, ACE), and beta (Bray-Curtis, weighted UniFrac) diversity metrics were calculated in QIIME. A one-way analysis of similarity (ANOSIM) was used to determine differences between groups. Differences between the TM groups and the two other groups were calculated using STAMP [18]. Differences in alpha diversity and relative abundance of taxa among different groups were analyzed using the Kruskal-Wallis rank-sum test in R. Differences between all three groups were determined using Welch's test corrected for a false discovery rate (FDR) according to Benjamini-Hochberg procedure. PICRUSt v.1.0.1 was used to assess the metabolic potential of the gut microbiota [19].

\section{Results}

\section{Microbiota Diversity}

We obtained a total of 766,114 high-quality sequences: between 33,247 and 47,873 valid sequences for each sample (Table S1). The rarefaction curves for the OTUs detected showed that the number of OTUs increased with the depth of sequencing. The final curve became stable, implying that the amount of sequencing data is somewhat reasonable (Fig. S1). Sequences were classified into 20 phyla, 43 classes, 67 orders, 113 families, and 234 genera (Table S2). There were 1470 OTUs shared by all three groups, and 1774, 949, and 1200 unique OTUs in the OM, TH, and ON groups, respectively (Fig. 1). The number of OTUs increased with age. The Prevotella, Butyrivirio, Clostridium.

There were significant differences in alpha diversity (ACE, Chao1, Shannon, and Simpson indices) between the TM and ON groups $(P<0.05)$, as well as the TM and TH groups $(P<0.05)$ (Fig. 2$)$. There was not a significant difference in the alpha diversity of the $\mathrm{ON}$ and $\mathrm{TH}$ groups $(P>0.05)$. A principal coordinates analysis of the weighted Unifrac distance showed that samples clustered according to age. The $\mathrm{ON}$ and $\mathrm{TH}$ samples were more similar than the TM samples with any other groups (Fig. 3). The composition difference was also determined by Partial Least Squares Discriminant analysis (Fig. S1) as well as analysis of similarities (ANOSIM) using weighted UniFrac distances $(R=0.5202$, $P=0.001)$.

\section{Characterization of the Microbiota}

At the phylum level, Gut bacterial communities showed clear age differences in the Firmicutes (TH 59.27\%, ON $60.62 \%$, TM 56.39\%), Bacteroidetes (TH 24.03\%, 19.45\%, TM 11.60\%), Verrucomicrobia (TH 8.09\%, ON 14.18\%, TM 1.85\%), and Proteobacteria (TH 3.22\%, 2.39\%, TM $17.28 \%$ ) abundances (Fig. 4a, Table S3). The relative abundance ratios of Proteobacteria and Actinobacteria were higher in the 2-month-old group than other groups $(P<0.05)$. The microbiota of the $\mathrm{ON}$ and $\mathrm{TH}$ groups was dominated by Firmicutes, Bacteroidetes, and Verrucomicrobia $(P<0.05)$. At the genus level, the relative abundances 
Fig. 1 Venn diagram showing the overlap of OTUs across groups. $O N 1$ year old, $T H 3$ years old, TM 2 months old

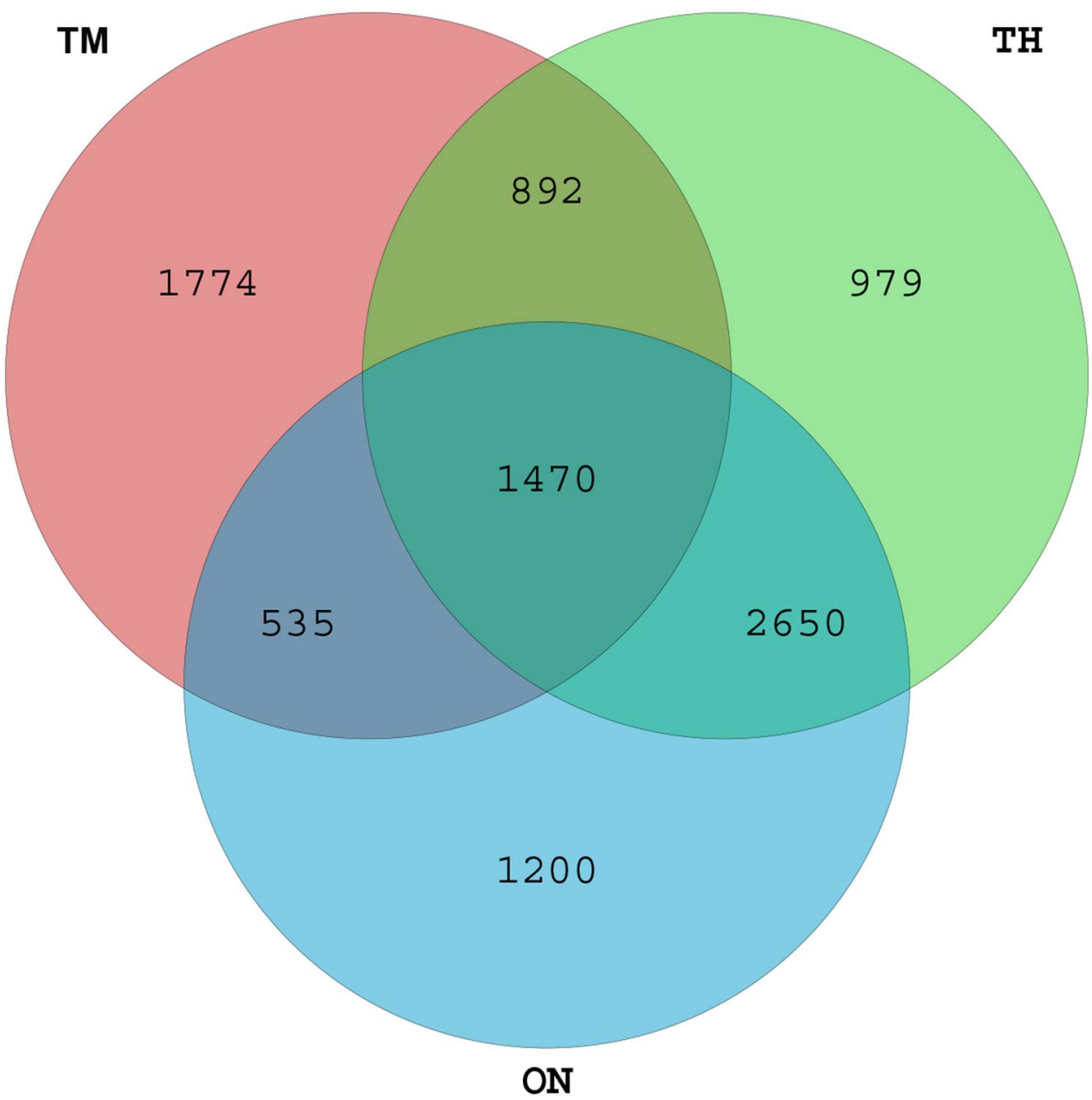

of Escherichia-Shigella (13.45\%), Blautia (5.43\%), and Alistipes (5.0\%) were highest in the TM group; while Ruminococcaceae_UCG-005 (ON 12.8\%, TH 10.0\%), Akkermansia (ON 13.9\%, TH 7.7\%), and Christensenellaceae_R-7_ group (ON 10.5\%, TH 8.3\%) were the most abundant in the TM and ON groups (Fig. 4b, Table S4). One sample in the TM group, TM5, had a markedly different community structure from the rest of the samples in that group (Fig. 5). Community structure in the ON group was similar to that of the TH group. In comparison with TM group, Christensenellaceae_R-7_group, Ruminococcaceae_UCG -005, Ruminococcaceae_UCG-010, Akkermansia, and Prevotellaceae_UCG-003 increased significantly in the $\mathrm{ON}$ and TH groups $(P<0.05)$. However, The relative abundances of Streptococcus, Blautia, Fusobacterium, and Bifidobacterium were lower in the $\mathrm{ON}$ and $\mathrm{TH}$ groups than in the TM group $(P<0.05)$ (Fig. 6, Table S5).

\section{Predicted Functions of Microbiota}

To estimate changes in the metabolic potential of the gut microbiota with age, we applied the PICRUSt algorithm.
The metabolic capacities of the $\mathrm{ON}$ and TH groups were similar, while the TM group was distinct from the other two groups (Fig. S3). There were significant differences between the TM and TH groups in the predicted abundance of pathways related to immune system; folding, sorting, and degradation; replication and repair; and translation and immune system diseases $(P<0.05)$. The mean relative abundance of immune system disease-related pathways was higher in the TM group; while the relative abundance of immune system related pathways was lower in the TM group (Fig. 7).

\section{Discussion}

Previous studies have described the longitudinal segregation of the gut microbiota in Bactrian camel [12]; however, the core gut microbiome of the Bactrian camel in different ages still remain unclear. To the best of our knowledge, in this study, we analyzed the bacterial diversity and abundance of fecal contents of Bactrian camel in different ages with high-throughput sequencing. In mammals, the distribution of microbes is influenced by host genotype, gender, and age 

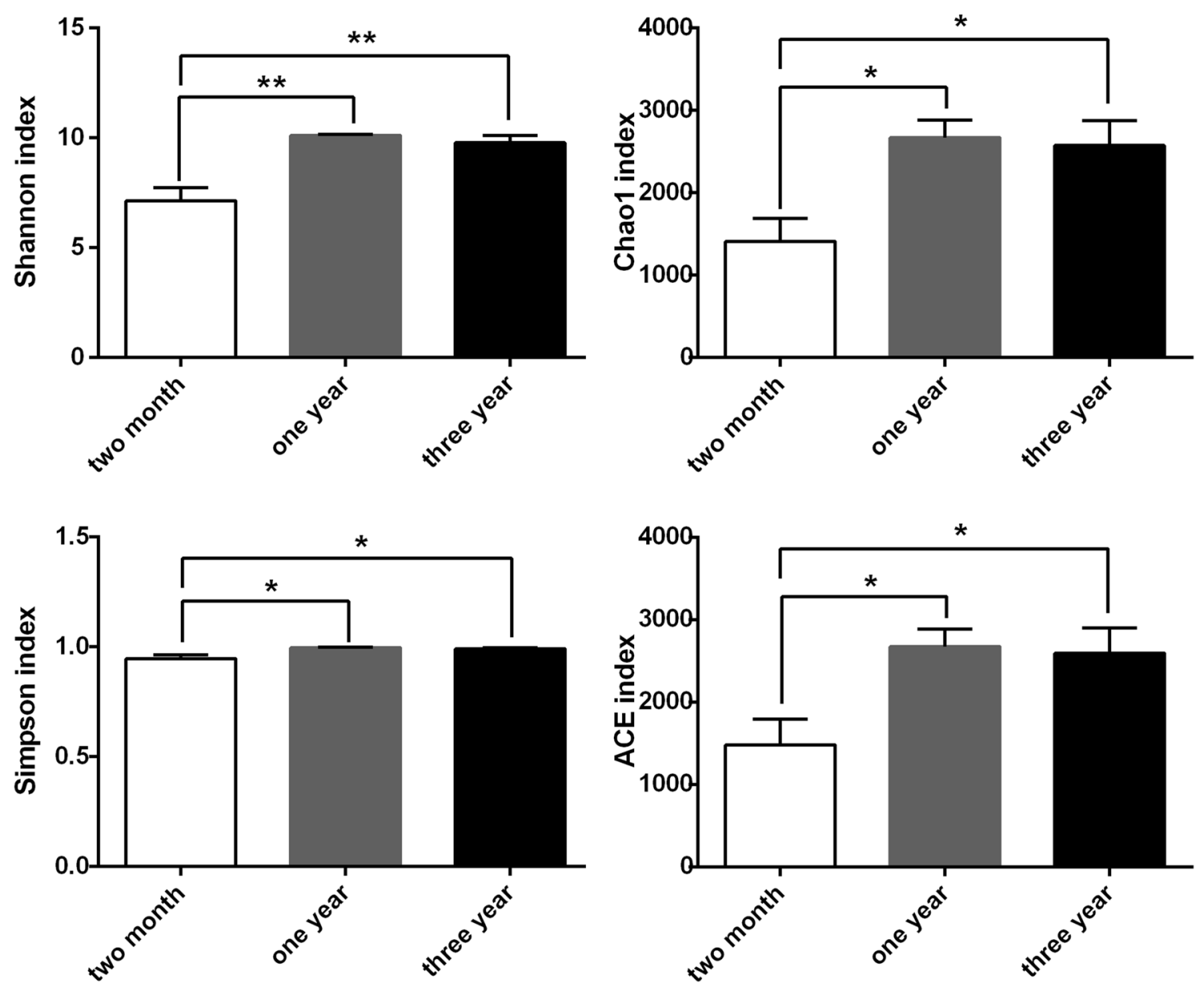

Fig. 2 Differences in alpha diversity between the three groups. $* P<0.05$, $* * P<0.01$

$[20,21]$. In this study, we show that the composition of the fecal microbiota changes during different ages. Firmicutes, Bacteroidetes revealed the most abundant taxa in the fecal microbiota. The abundance of these two major phyla was largely consistent with mature camel rumen [10]. At the genus level, Prevotella, Butyrivirio, Spriochaetes, Verrucomicrobia, Succinivibria, Clostridium were common in the core microbiome of camel rumen [10, 22]. However, the taxonomic groups represented within the Bactrian camel feces were not similar to those previously observed in the rumen of camels. Ruminococcaceae_UCG-005, Akkermansia, and Christensenellaceae_R-7_group were the most abundant in the mature fecal microbiota.

The richness and diversity of the microbiota increase with age $[23,24]$. We observed differences in microbiota composition between the TM and ON/TH groups. By 1 year of age, the $\alpha$-diversity (total OTU counts) approached adult levels and the $\beta$-diversity was similar to the TH group (Figs. 1, 2, 4). It is an indication that as Bactrian camel reached 1 year of age the microbiota gradually stabilized towards an adultlike state, which suggests that the 1-year-old Bactrian camel gut microbiome has many functional attributes of the adult microbiome.

Interestingly, at the phylum level, the increase in the relative abundance of Firmicutes, Bacteroidetes, and Verrucomicrobia that we observed, along with the decline in Proteobacteria and Actinobacteria and increase in Firmicutes and Bacteroidetes, is in agreement with previous studies [25, 26]. Microbiota diversity was primarily driven by diet. The dominant taxa in the TM group were Firmicutes and Proteobacteria, compared to Firmicutes, Bacteroidetes, and Verrucomicrobia for the $\mathrm{ON}$ and $\mathrm{TH}$ groups. Previous study has been reported that the Firmicutes/Bacteroidetes ratio changes with age [27]; Microbiota diversity was primarily driven by diet. The dominant taxa in the TM group were Firmicutes and Proteobacteria, compared to Firmicutes, Bacteroidetes, and Verrucomicrobia for the $\mathrm{ON}$ and TH groups. Previous studies have reported that the ratio of Firmicutes/ Bacteroides changes with age [28], which is consistent with this report. Interestingly, the relative abundance ratios of Proteobacteria and Actinobacteria were higher in the TM group. Proteobacteria is one of the earliest colonizers and 
Fig. 3 Principal coordinates analysis of weighted UniFrac distances. $O N 1$ year old, $T H 3$ years old, TM 2 months old
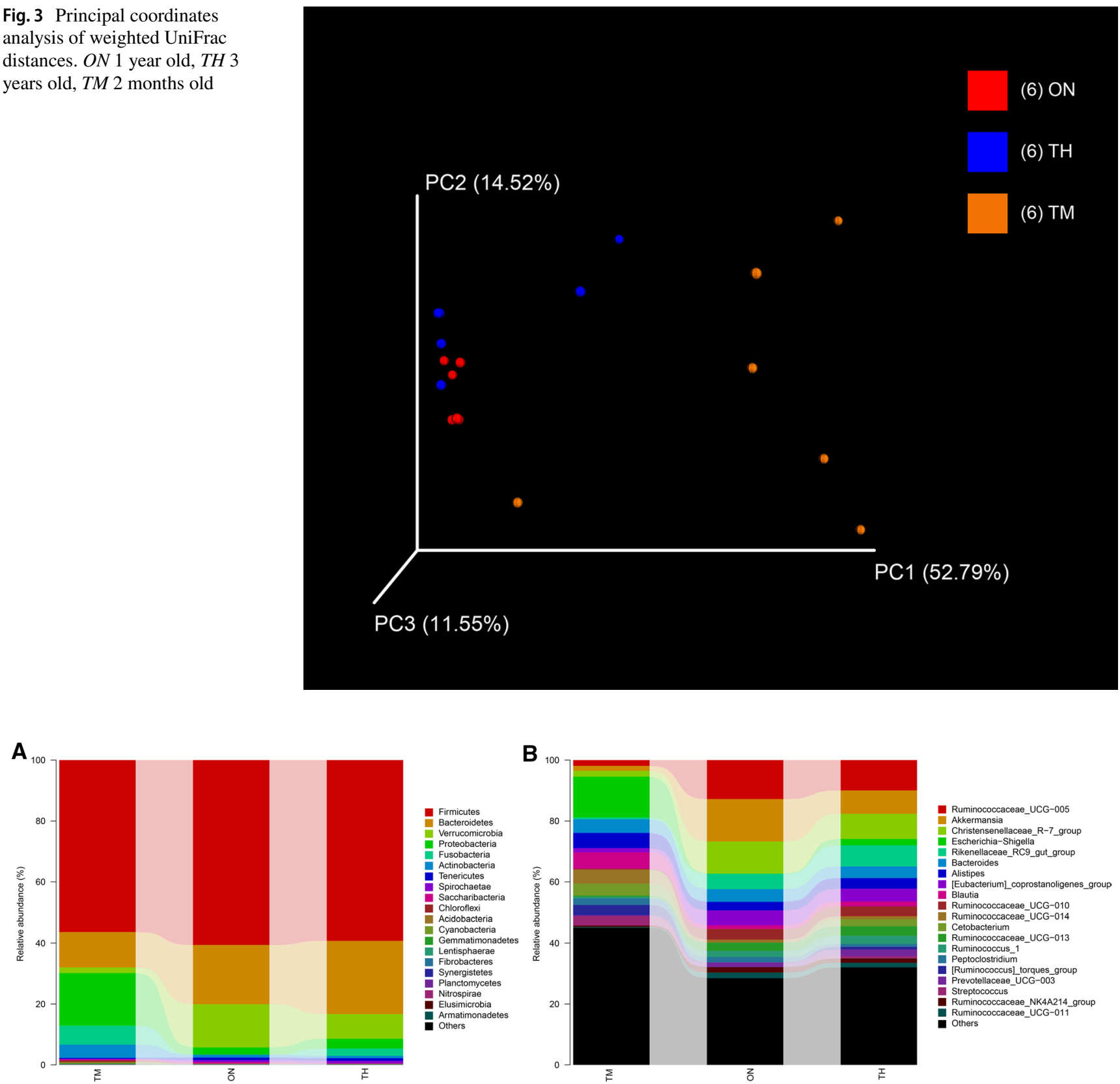

Fig. 4 Microbial composition of different samples. a Taxa assignments at phylum level. b Taxa assignments at genus level. ON 1 year old, TH 3 years old, TM 2 months old

main members in the neonatal. It is conductive to homeostasis of the anaerobic environment of the GI tract gut [29]. Moreover, Actinobacteria coordination with one partner or host can easily be translated into pathogenic interactions with another [30].

At the genus level, Escherichia-Shigella, Blautia, and Alistipes were the abundant microbiota in the TM group. It has recently been reported that Blautia can exhibit beneficial anti-inflammatory effects [31]. Previous studies have described the dominance of Escherichia-Shigella in young calves and first-day human meconium [14, 24]. In addition to Escherichia-Shigella, Streptococcus, Blautia, and Fusobacterium had increased abundances in the TM group $(P<0.05)$ (Fig. 5). The proportion of some intestinal microbiota changes with age. Compared with camel calves, the proportion of the ratio of Christensenellaceae_R-7_ group, Ruminococcaceae_UCG-005, Ruminococcaceae_ UCG-010, were dominant in adult camels. Ruminococcaceae and Christensenellaceae regarded as potential beneficial bacteria because they participated in the positive 
Fig. 5 Heatmap of genus level relative abundances. $O N 1$ year old, $T H 3$ years old, $T M 2$ months old

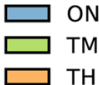

\section{TM}

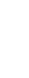
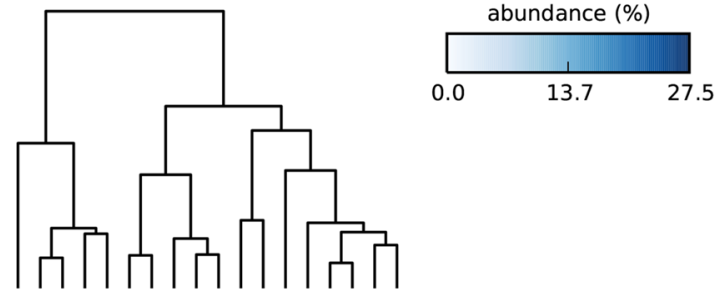
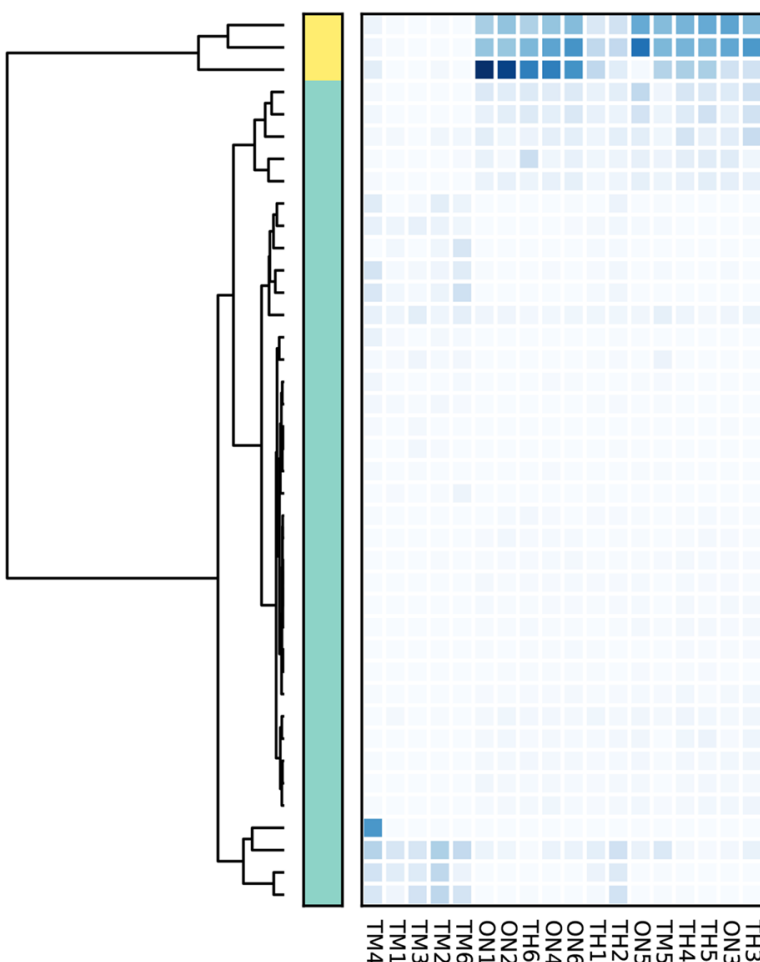

Christensenellaceae R-7 group Ruminococcaceae_ŪCG-0̄05 kkermansia

Ruminococcaceae UCG-010 Ruminococcaceae-UCG-013 Ruminococcus 1

Prevotellaceae UCG-003

Ruminococcaceae UCG-011

[Eubacterium] rectale_group

[Eubacterium] rec

Lachnoclostridium
Subdoligranulum

Clostridium_sensu_stricto_1

Butyricicocc̄us

Lachnospiraceae UCG-008

Erysipelatoclostrídium

[Ruminococcus]_gauvreauii_group

Arthrobacter

Ruminiclostridium_5

Gemmatimonas

Moryella

Sphingomonas

Butyricimonas

Phocaeicola

Barnesiella

[Eubacterium]_nodatum_group

Prevotella 1

Lachnoclostridium 10

Ruminiclostridium-1

[Eubacterium] brachy_group

Family_XIII_UCG-002

Lachnospirāceae AC2044 group

Family_XIII_AD3011_group

dgA-1'__gut_group

Candidatus Soleaferrea

Prevotellacéae UCG-004

Ruminococcacēae_UCG-009

usobacterium

Blautia

Streptococcus

[Ruminococcus]_torques_group

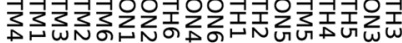
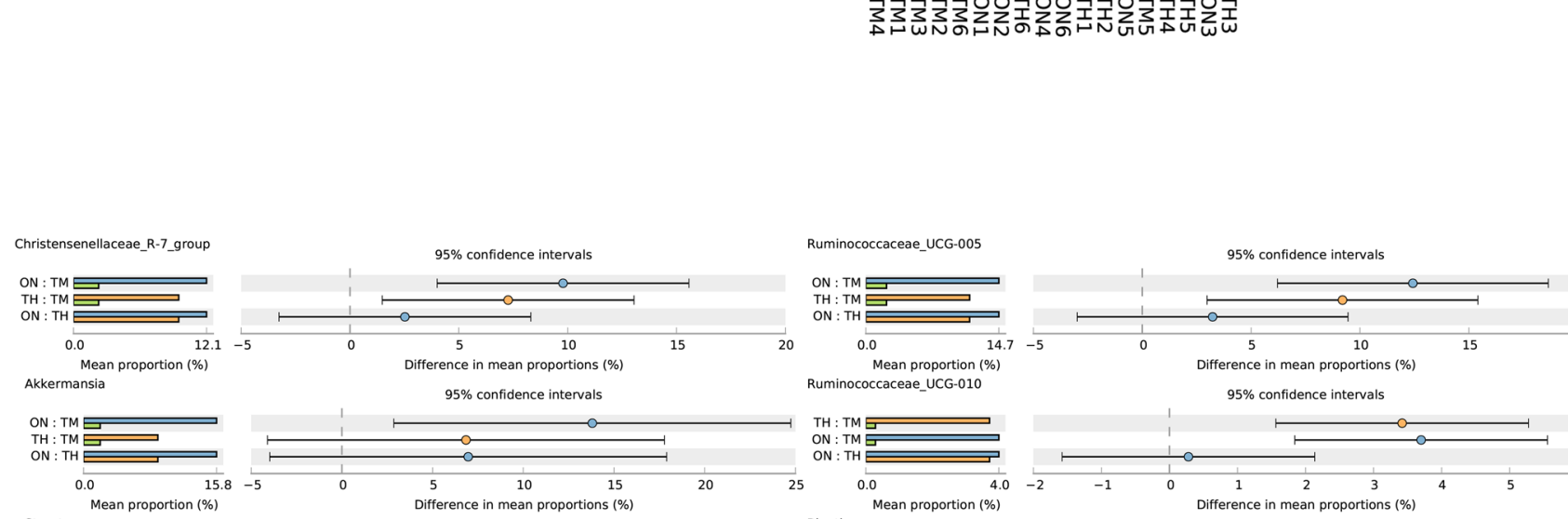

Mean proportion (\%)

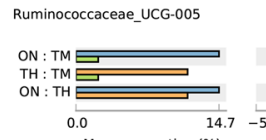

Mean proportion (\%) Ruminococcaceae_UCG-010

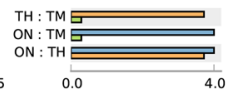

Mean proportion $(\%)$ Blautia

$95 \%$ confidence intervals

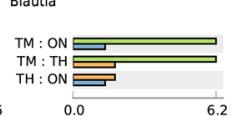

0.0
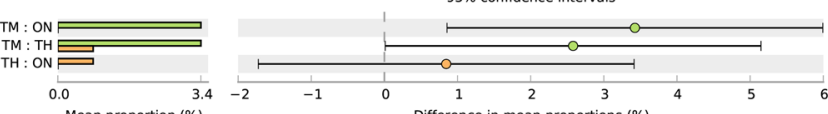

Bifidobacterium
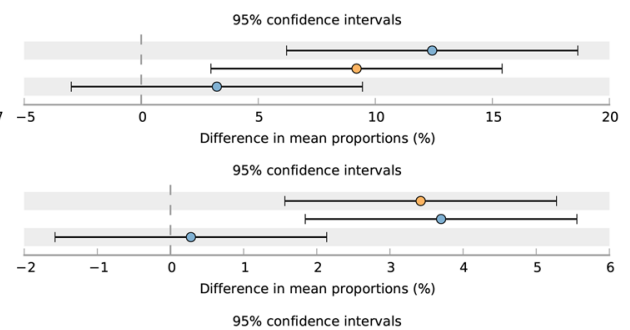

Mean proportion (\%)

Fusobacterium

$95 \%$ confidence intervals
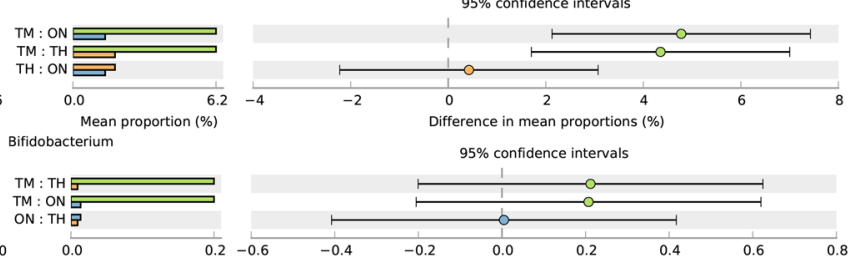

Fig. 6 An extended error bar plot indicating the differences in mean abundance of taxa in the three groups. $O N 1$ year old, TH 3 years old, TM 2 months old 


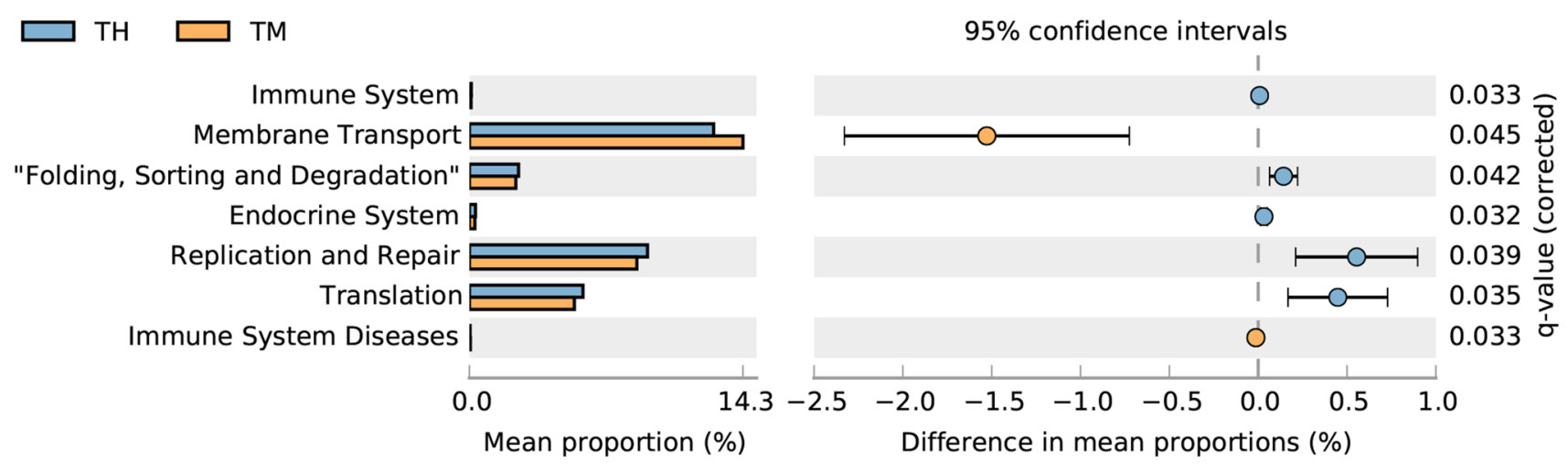

Fig. 7 Predicted KEGG pathways for the TM and TH groups determined using PICRUSt. TH 3-year-old group, TM 2-month-old group

regulation of the intestinal environment and linked to immunomodulation and healthy homeostasis [32, 33]. It suggests that health state is also related to ages. The significant differences for some KEGG categories, such as Immune system and immune system diseases, were also higher in the 2-month-old compared with other camels. The composition of the microbial community at 2 months of age may be heavily influenced by weaning, in which diarrhea is easy to occur. This shift from a milk- to a plantbased diet could also explain the differences in metabolic potential. The intestinal mucosal developmental immaturity and the external environment may make the camel calves more susceptible to pathogen invasion [34]. Previous study has showed that intestinal microflora was closely related to diarrhea [35].

In conclusion, our data suggest that microbiota composition may be due to changes in the diet and/or physiological changes associated with age. Gut microbiota structure is similar between the $\mathrm{ON}$ and $\mathrm{TH}$ groups, suggesting that maturation may occur by 1 year of age. However, we do not have enough time points to determine precise population dynamics. Bacterial colonization starts at birth and plays important roles in host growth and immune development [36]. A stable commensal community protects the host from invasive pathogens [3]. The establishment of stable microbial communities has an important role in the induction of homeostatic mechanisms [37]. Future work should examine the development of an adult microbiota as a basis for understanding how diet and host microbiota impact the development of the Bactrian camel.

Acknowledgements This work was supported by grants from Inner Mongolia Autonomous Region Science and Technology Innovation Guide Project (KCMS2018048), Natural Science Foundation Project of Inner Mongolia (2018BS03017), Double-class discipline innovation team building (NDSC2018-14), High-level talents introduce scientific research start-up project (NDYB2017-28), and Major special project of the Inner Mongolia Autonomous Region. We thank Personal
Biotechnology Co., Ltd. (Shanghai China) for their sequencing service and the native English speaking scientists of Elixigen Company (Huntington Beach, California) for editing our manuscript.

Author Contributions JRMT designed the experiments. JH, LH, and GwL participated in data analysis. JH, $\mathrm{LH}, \mathrm{KO}$, GwL, and FcG participated in the sample collection. JH wrote the paper. LY and LM advised on the manuscript content and reviewed the manuscript. All authors read and approved the final manuscript.

Data Availability Illumina sequence reads have been deposited under the NCBI SRA accession number SRP180948.

\section{Compliance with Ethical Standards}

Conflict of interest The authors declare no conflict of interest.

Open Access This article is distributed under the terms of the Creative Commons Attribution 4.0 International License (http://creativeco mmons.org/licenses/by/4.0/), which permits unrestricted use, distribution, and reproduction in any medium, provided you give appropriate credit to the original author(s) and the source, provide a link to the Creative Commons license, and indicate if changes were made.

\section{References}

1. Flint HJ, Scott KP, Louis P, Duncan SH (2012) The role of the gut microbiota in nutrition and health. Nat Rev Gastroenterol Hepatol 9(10):577-589

2. Malmuthuge N, Li M, Goonewardene LA, Oba M, Guan LL (2013) Effect of calf starter feeding on gut microbial diversity and expression of genes involved in host immune responses and tight junctions in dairy calves during weaning transition. J Dairy Sci 96(5):3189-3200

3. Kamada N, Chen GY, Inohara N, Núñez G (2013) Control of pathogens and pathobionts by the gut microbiota. Nat Immunol 14(7):685-690

4. Chung H, Pamp SJ, Hill JA, Surana NK, Edelman SM, Troy EB, Reading NC, Villablanca EJ, Wang S, Mora JR (2012) Gut immune maturation depends on colonization with a host-specific microbiota. Cell 149(7):1578-1593 
5. Funkhouser LJ (2013) Mom knows best: the universality of maternal microbial transmission. PLoS Biol 11(8):e1001631

6. Wu HJ, Wu E (2012) The role of gut microbiota in immune homeostasis and autoimmunity. Gut Microbes 3(1):4-14

7. Russell JB, Rychlik JL (2001) Factors that alter rumen microbial ecology. Science 292(5519):1119-1122

8. Eugene R, Gil S, Ilil A, Ilana ZR (2010) The evolution of animals and plants via symbiosis with microorganisms. Environ Microbiol Rep 2(4):500-506

9. Mohandesan E, Fitak RR, Corander J, Yadamsuren A, Chuluunbat B, Abdelhadi O, Raziq A, Nagy P, Stalder G, Walzer C (2017) Mitogenome sequencing in the genus Camelus reveals evidence for purifying selection and long-term divergence between wild and domestic Bactrian camels. Sci Rep 7(1):9970

10. Gharechahi J, Zahiri HS, Noghabi KA, Salekdeh GH (2015) Indepth diversity analysis of the bacterial community resident in the camel rumen. Syst Appl Microbiol 38(1):67-76

11. Gharechahi J, Salekdeh GH (2018) A metagenomic analysis of the camel rumen's microbiome identifies the major microbes responsible for lignocellulose degradation and fermentation. Biotechnol Biofuels 11(1):216

12. He J, Yi L, Hai L, Ming L, Gao W, Ji R (2018) Characterizing the bacterial microbiota in different gastrointestinal tract segments of the Bactrian camel. Sci Rep 8(1):654

13. Sun ML, Kim N, Ji HP (2018) Comparative analysis of ileal and cecal microbiota in aged rats. J Cancer Prev 23(2):70-76

14. Alipour MJ, Jalanka J, Pessamorikawa T, Kokkonen T, Satokari R, Hynönen U, Iivanainen A, Niku M (2018) The composition of the perinatal intestinal microbiota in cattle. Sci Rep 8(1):10437

15. Gu S, Chen D, Zhang JN, Lv X, Wang K, Duan LP, Nie Y, Wu XL (2013) Bacterial community mapping of the mouse gastrointestinal tract. PLoS ONE 8(10):e74957

16. Caporaso JG, Kuczynski J, Stombaugh J, Bittinger K, Bushman FD, Costello EK, Fierer N, Peña AG, Goodrich JK, Gordon JI (2010) QIIME allows analysis of high-throughput community sequencing data. Nat Methods 7(5):335-336

17. Quast C, Pruesse E, Yilmaz P, Gerken J, Schweer T, Yarza P, Peplies J, Glöckner FO (2013) The SILVA ribosomal RNA gene database project: improved data processing and web-based tools. Nucleic Acids Res 41(Database issue):590-596

18. Parks DH, Beiko RG (2010) Identifying biologically relevant differences between metagenomic communities. Bioinformatics 26(6):715-721

19. Langille MGI, Zaneveld J, Caporaso JG, Mcdonald D, Dan K, Reyes JA, Clemente JC, Burkepile DE, Thurber RLV, Knight R (2013) Predictive functional profiling of microbial communities using 16S rRNA marker gene sequences. Nat Biotechnol 31(9):814-821

20. Pfefferle PI, Renz H (2014) The mucosal microbiome in shaping health and disease. F1000prime Rep 6:11

21. Berding K, Holscher HD, Arthur AE, Donovan SM (2018) Fecal microbiome composition and stability in 4- to 8-year old children is associated with dietary patterns and nutrient intake. J Nutr Biochem 56:165-174

22. Bhatt VD, Joshi CG, Dande SS, Patil NV (2013) Molecular analysis of the bacterial microbiome in the forestomach fluid from the dromedary camel (Camelus dromedarius). Mol Biol Rep 40(4):3363-3371
23. Yatsunenko T, Rey FE, Manary MJ, Trehan I, Dominguezbello MG, Contreras M, Magris M, Hidalgo G, Baldassano RN, Anokhin AP (2012) Human gut microbiome viewed across age and geography. Nature 486(7402):222-227

24. Bäckhed F, Roswall J, Peng Y, Feng Q, Jia H, Kovatchevadatchary P, Li Y, Xia Y, Xie H, Zhong H (2015) Dynamics and stabilization of the human gut microbiome during the first year of life. Cell Host Microbe 17(5):690-703

25. Muinck EJD, Trosvik P (2018) Individuality and convergence of the infant gut microbiota during the first year of life. Nat Commun 9(1):2233

26. Wenjing Z, Yapeng W, Shuyun L, Jiaojiao H, Zhengxiao Z, Chuan H, Jinmei D, Jun W, Huijuan W, Weibing F (2015) The dynamic distribution of porcine microbiota across different ages and gastrointestinal tract segments. PLoS ONE 10(2):e0117441

27. Mariat D, Firmesse O, Levenez F, Guimarăes V, Sokol H, Doré J, Corthier G, Furet J-P (2009) The Firmicutes/Bacteroidetes ratio of the human microbiota changes with age. BMC Microbiol 9(1):123-130

28. Zapata HJ, Quagliarello VJ (2015) The microbiota and microbiome in aging: potential implications in health and age-related diseases. J Am Geriatr Soc 63(4):776-781

29. Moon CD, Young W, Maclean PH, Cookson AL, Bermingham EN (2018) Metagenomic insights into the roles of Proteobacteria in the gastrointestinal microbiomes of healthy dogs and cats. Microbiologyopen 7(5):e00677

30. Miao V, Davies J (2010) Actinobacteria: the good, the bad, and the ugly. Antonie Van Leeuwenhoek 98(2):143-150

31. Jenq RR, Ying T, Devlin SM, Ponce DM, Goldberg JD, Ahr KF, Littmann ER, Ling L, Gobourne AC, Miller LC (2015) Intestinal blautia is associated with reduced death from graft-versus-host disease. Biol Blood Marrow Transplant 21(8):1373-1383

32. Kong F, Hua Y, Bo Z, Ning R, Ying L, Zhao J (2016) Gut microbiota signatures of longevity. Curr Biol 26(18):R832-R833

33. Shang Q, Shan X, Cai C, Hao J, Li G, Yu G (2016) Dietary fucoidan modulates the gut microbiota in mice by increasing the abundance of Lactobacillus and Ruminococcaceae. Food Funct 7(7):3224-3232

34. Andrés S, Jiménez A, Sánchez J, Alonso JM, Gómez L, López F, Rey J (2007) Evaluation of some etiological factors predisposing to diarrhoea in lambs in "La Serena" (Southwest Spain). Small Rumin Res 70(2):272-275

35. Türkyılmaz S, Eskiizmirliler S, Tunaligil S, Bozdogan B (2014) Identification, characterization and molecular epidemiology of Escherichia coli isolated from lamb and goat kids with diarrhoea. Acta Veterinaria Brno 82(4):357-362

36. Power ML, Quaglieri C, Schulkin J (2017) Reproductive microbiomes: a new thread in the microbial network. Reprod Sci 24(14):1482-1492

37. Renz H, Brandtzaeg P, Hornef M (2012) The impact of perinatal immune development on mucosal homeostasis and chronic inflammation. Nat Rev Immunol 12(1):9-23

Publisher's Note Springer Nature remains neutral with regard to jurisdictional claims in published maps and institutional affiliations. 